\title{
Celebrating invention
}

\section{Edwin Southern}

Scientists seeking explanations for what they see in the natural world usually start with an idea, a hypothesis. In the biological sciences, this first step will almost always be followed by an experimental test. Often the tools are to hand and experiments can go ahead. But what if they are not? Often, progress is stalled; however, sometimes the scientist turns inventor and gets on with devising a new method to fit the task, leaving the kits in the cupboard. If this activity does lead to a successful new method, it is rarely the end of the matter.

The topics in this Milestones in DNA Technologies series show that methods born of the need to answer specific questions often go on to be used in different applications from those envisaged by the inventor. Some methods, such as PCR, are so simple, elegant and useful that they rapidly permeate the whole of the field.

Other methods have such power that they can drive biological science in completely new directions, altering the way in which scientists think and operate.

A prime example of such far-reaching effect is Sanger sequencing, which gave birth to genomics and to an information-based approach to biology: experiment precedes hypothesis. We sequence genomes because we can, and ask questions later. Simpler analytical techniques brought increased power to genetic studies; human genetics can now address inherited diseases in ways that were not conceivable before the advent of DNA-based genotyping methods. Another major revolution in biology grew from the bold vision that the genes from one species could function in another. From this vision sprang all of the methods for cloning DNA and for the genetic transformation of organisms.

\section{A prime example of such} far-reaching effect is Sanger sequencing, which gave birth to genomics and to an information-based approach to biology: experiment precedes hypothesis.
The immediate future might be similarly unforeseen by the inventor. The steps that must be taken to solve technical challenges might lead the inventor into an unfamiliar discipline. It is instructive to look at the diversity of components that were assembled by Sanger into his methods for sequencing complex nucleic acids - novel applications of electrophoresis, new and artificial substrates for DNA polymerase, and new ways of data handling in computers. In these ancillary areas there is often scope for improvement, for other inventors to stand on giant's shoulders. Sanger sequencing saw fluorescence labelling replace radiolabelling, and capillary electrophoresis replace slab gels, which further allowed high-throughput automation and the development of the whole field of bioinformatics, which grew from methods for handling sequence information. Four of the milestones in this collection are devoted to extensions and improvements of Sanger's original invention.

The importance and value of experimental methods has not always been appreciated as it is today. There is, perhaps, despite all the achievements, a lingering sense that theory is somehow better than experiment, and that science sits on a higher plane than invention. Perhaps there is a sense that the doing of experiments needs less intelligence than the guiding and interpreting of them. I would argue against this position. Clearly, devising a new method is no less intellectually demanding than the process of making discoveries. It requires conviction and confidence on the part of the inventor that any problems can be surmounted, as well as the audacity to venture into different disciplines when needed. For some, this is part of the appeal, the stimulus to the imagination that fuels lateral thinking and innovation.

The divide between theory and experiment, and between discovery and invention, is reflected in journal publication. The experimental section, when there is one, is often printed in small type, or the experimental details are relegated to figure captions or footnotes. It is a paradox that journals that specialize in method development are low in the ratings. They hold some treasures: the two most cited papers of the past 20 years were published in Analytical Biochemistry. Moreover, the rewards are high for those lucky enough to have an aptitude and enthusiasm for method development: major prizes have gone to inventors, and fame has often followed; there is money to be made through commercialization; and, above all, the inventor has the satisfaction of seeing the results and discoveries engendered by a new method. Against this background, it is encouraging to see the Nature Publishing Group, through Nature Methods and the present collection assembled by several journals, giving a higher profile to technology development. It is particularly pleasing to join in this celebration of the great contributions made by DNA technologies. These are the methods that revealed to us the common molecular basis of all living organisms.

Edwin Southern is Professor of Biochemistry at the Department of Biochemistry, University of Oxford, South Parks Road, Oxford OX1 30U, UK. e-mail: ed.southern@bioch.ox.ac.uk

doi: $10.1038 / \operatorname{nrg} 2248$ 